\title{
Tejidos de Clío construidos en regiones que transforman el espacio a través del tiempo: relaciones entre la enseñanza y la Historia regional*
}

\section{Resumen}

El presente artículo hace parte de los resultados de la investigación "La formación de la Conciencia Histórica a partir del estudio de la Historia regional" con la intención de aportar a los procesos de formación docente en este campo. Se realiza un acercamiento concreto a la enseñanza de la Historia Regional tanto como una postura de pensar la Historia, así como una metodología para su estudio. El desarrollo de la investigación se hizo desde la Teoría Fundamentada, con una integración entre el trabajo teórico y práctico como eje para proponer una teoría sustantiva. Se concluye que la región histórica es una forma de desarrollar procedimientos propios de la historia y que otorgan al docente de la educación básica y media, la posibilidad de relacionar la región en las aulas de clase desde posibilidades de estudios dinámicos sobre las relaciones de frontera y la relación de las comunidades en las mismas.

\section{Palabras clave}

Tesauro: Historia, enseñanza, ciencias sociales.

Autor: Historia regional, región.

Referencia bibliográfica para citar este artículo: Loaiza Zuluaga, Yadaldez Eder y Pantoja Suárez, Paula Tatiana. "Tejidos de Clío construidos en regiones que transforman el espacio a través del tiempo: relaciones entre la enseñanza y la Historia regional". Anuario de Historia Regional y de las Fronteras 26.1 (2021): 251-272.

Yasaldez Eder Loaiza Zuluaga: Doctor en Ciencias de la Educación de la Universidad Pedagógica y Tecnológica de Colombia. Profesor titular del Departamento de Estudios Educativos y director del Doctorado en Educación de la Universidad de Caldas. Líder del Grupo de Investigación Maestros y Contextos. Código ORCID: 0000-0003-4215-2267. Correo electrónico: yasaldez@ucaldas.edu.co.

Paula Tatiana Pantoja Suárez: candidata a Doctora en Educación por la Universidad de Caldas. Magister en Educación por la Universidad de Caldas. Docente del Departamento de Historia de la Universidad de Caldas. Código ORCID: 0000-0003-0093-4164. Correo electrónico: paula.pantoja@ucaldas.edu.co.

\footnotetext{
* Este artículo es el resultado del trabajo desarrollado a través del Programa de Investigación Reconstrucción del Tejido Social en Zonas de Posconflicto en Colombia. Cósigo SIGP: 57579 con el proyecto de investigación Alianza inter-institucional, multidisciplinar, nacional e internacional en el aumento de la calidad educativa , científica, innovadora y productiva de las instituciones educativas de educación superior. El cual fue financiado en el marco de la convocatoria Colombia Científica, contrato número FP44842-213-2018.
} 


\title{
Clío Networks built in regions that transform space through time: relationships between teaching and the regional History
}

\begin{abstract}
This article is part of the results of the research "The formation of Historical Awareness from the study of regional History" and attempts to contribute to the processes of teacher training in this field. A concrete approach was made to the teaching of Regional History as well as a position of thinking about History, as well as a methodology for its study. The development of the research was done from Grounded Theory, with an integration between theoretical and practical work as the axis to propose a substantive theory. It is concluded that the historical region is a way of developing procedures typical of history and that they give the teacher of basic and secondary education, the possibility of relating the region in the classrooms from possibilities of dynamic studies on border relations and the relationship of the communities in them.
\end{abstract}

Thesaurus: History, Teaching, Social Sciences

Keywords: Regional History, Region.

\section{Tecidos de Clío construídos em regiões que transformam o espaço ao longo do tempo: relações entre o ensino e a História regional}

\section{Resumo}

Este artigo é parte dos resultados da pesquisa "A formação da Consciência Histórica a partir do estudo da História regional" e procura contribuir com os processos de formação de professores nesta área. Faz-se uma abordagem concreta do ensino da História Regional e também uma postura de pensar a História, bem como uma metodologia para o seu estudo. O desenvolvimento da pesquisa foi feito a partir da Teoria Fundamentada nos dados, tendo a integração entre o trabalho teórico e prático como eixo para a proposição de uma teoria substantiva. Conclui-se que a região histórica é uma forma de desenvolver procedimentos típicos da história e que dão ao professor do ensino fundamental e médio, a possibilidade de relacionar a região nas aulas a partir de possibilidades de estudos dinâmicos sobre relações de fronteira e a relação das comunidades nelas.

Tesauro: História, ensino, ciências sociais.

Palavras Chave: História regional, região. 


\section{Construcción de sentido: la teoría emergente ${ }^{1}$}

\subsection{Reflexión necesaria para abordar el problema de la región y la Historia Regional}

¿Qué es la región?, pero la región ¿en qué contexto?, la región como parte de un país o la región como parte de un continente o la región como parte de un todo; todo en este caso, puede ser el mundo. Estas palabras de un docente de la Licenciatura en Ciencias Sociales de la Universidad de Caldas son el inicio de una reflexión por definir qué es región y la complejidad de dicha labor en aras de las regiones como eje de estudio para la Historia y su enseñanza. Para Van Young "las regiones son como el amor, no pueden definirse, pero al verlas se sabe que son. Este tipo de reflexiones enmarcan en la definición de región una situación compleja, pero enriquecedora por las múltiples posturas que la construyen".?

Sobre el concepto de región, expone Van Young que, en su forma más útil es la "espacialización" de una relación económica. Acotando además que, una definición funcional muy simple sería la de un espacio geográfico con una frontera que lo delimita, la cual estaría determinada por el alcance efectivo de algún sistema cuyas partes interactúan más entre sí que con los sistemas externos. Por un lado, la frontera no necesita ser impermeable $\mathrm{y}$, por otro, no es necesariamente congruente con las divisiones políticas o administrativas más familiares y fácilmente identificables, o aun con los rasgos topográficos; en tal sentido, es necesario que los estudios que hacen referencia al concepto de región tengan claridad, sobre el concepto en el que se enmarca; y a su vez evitar la confusión entre "regionalidad -la cualidad de ser de una región- y regionalismo, la identificación consciente, cultural, política y sentimental, que grandes grupos de personas desarrollan con ciertos espacios a través del tiempo". ${ }^{3}$

Para Milton Santos "las regiones son subdivisiones del espacio geográfico planetario, del espacio nacional o inclusive del espacio local, son espacios en conveniencia $\mathrm{y}$ en algunos casos funcionales del espacio mayor". ${ }^{4}$ Se constituyen como sistemas territoriales abiertos que en permanente interacción con otras regiones construyen su propia identidad económica, cultural, social y política. Las regiones en su relación entre lo interno y lo externo no ocurren por fuera del proyecto nacional, hacen parte de este y a su vez se constituyen en instrumento para el desarrollo de la nación.

\footnotetext{
${ }^{1}$ Es importante clarificar que, desde la perspectiva metodológica de la Teoría Fundamentada, los elementos del trabajo empírico realizado se relacionan de manera directa con los referentes teóricos de la propuesta investigativa. La recolección de la información se realizó a partir de entrevistas semiestructuradas en profundidad a una muestra de 10 docentes universitarios, 20 estudiantes del programa de Licenciatura en Ciencias Sociales de la Universidad de Caldas y 10 egresados del mismo programa. Este estudio, desde una perspectiva investigativa, no se encuentra realizado en otras universidades o centros de investigación del país; si bien se resalta el trabajo realizado por Álvaro Acevedo en una perspectiva historiográfica.

${ }^{2}$ Van Young, Eric, "Haciendo historia regional: Consideraciones metodológicas y teóricas" Región e historia en México (1700-1850): Métodos de análisis regional. Pedro Pérez Herrero. (México: Instituto de Investigaciones José Luis Mora Universidad Metropolitana, 1991) 99.

${ }^{3}$ Van Young, 102.

${ }^{4}$ Miltos Santos, Metamorfosis del espacio habitado (Barcelona: Editorial Oikos-tau, 1997).
} 
Por su parte, Etcheverry Bosier ${ }^{5}$ se refiere a la región como un territorio con una identidad histórica y una serie de valores que lo identifican con ciertas diferencias de otros espacios, así sean circundantes al mismo. Es interesante en este mismo autor el asunto de relacionar la ciudad-región como una construcción de mundo globalizado, en el cual la misma búsqueda por lo global ha llevado a la construcción y delimitación de espacios locales y el sentido sobre el territorio como un espacio de identidad y construcción de memoria. Estas ideas permiten ver el mundo como un todo y con diversas relaciones, formas, funciones, organizaciones, estructuras con sus más diversos niveles de interacción y contradicción. Las regiones aparecen como distintas regiones de la mundialización.

El concepto de piel social, ${ }^{6}$ establecido por Turner, se relaciona con la construcción y el sentido de identidad en la región, al definirse como "el atavío con que nos enfrentamos a la relación social, es el encuentro de la identidades..." todo ello está alimentado por una memoria y una historia incorporadas, que constituyen esta piel social de los sujetos y los convierten en sí mismos en una historia viva, independiente de su reconocimiento como seres histórico. Estos elementos tienen sentido para estudiantes de la Licenciatura en Ciencias Sociales a partir de reflexiones como la siguiente:

Puede que no sea determinante pero si es fundamental y se hace necesario en algunos estudios sociales sobre todo regionales comprender los procesos anteriores, para poder determinar y comprender lo que sucede hoy es necesario irse en la línea del tiempo hacia atrás para empezar a mirar qué fue los que sucedió antes en la región, los actores, los procesos, las estructuras y cómo fueron evolucionando a través del tiempo y eso puede marcar una tendencia hacia lo que posiblemente está sucediendo hoy en la región y así, darle una explicación al hoy desde el ayer. ${ }^{7}$

La región es entonces un espacio vivo históricamente y por ello se consolida como un objeto de estudio de la Historia, a partir de la reflexión sobre la memoria y la búsqueda por la misma, en aras de buscar y encontrar sentidos a las prácticas culturales que identifican a los grupos humanos y su sentido temporal, en la medida que son siempre producto de un pasado-presente que marca las alterativas de futuro de manera consciente o inconsciente.

\footnotetext{
${ }^{5}$ Etcheverry Boisier, "Territorio, Estado y Sociedad en Chile, la dialéctica entre la descentralización: entre la geografía y la gobernabilidad" (Tesis doctoral, Universidad de Alcalá. Alcalá de Henares, España, 2008) 42-46.

${ }^{6}$ La piel social es como el cuerpo- en el sentido de nuestras preguntas o incorporaciones como seres socialesque se constituye en el límite del individuo en cuanto a entidad biológica, psicológica y frontera del ser social. Nates, B., Jaramillo, P, Hernández, Más allá de la historia, sentidos de pertenencia, socialización y economía en el concepto de pueblo de los Andes. Manizales, Colombia: Editorial Universidad de Caldas. (2004) 20.

${ }^{7}$ Entrevista realizada por Paula Tatiana Pantoja a docentes en ejercicio y formación de la Licenciatura en Ciencias Sociales de la Universidad de Caldas. Manizales, agosto de 2012.
} 


\subsection{La región como objeto histórico}

El estudio de las regiones como un saber y objeto de estudio de la historia, es un asunto relativamente reciente ${ }^{8}$ el cual se desarrolló principalmente a partir de los estudios e investigaciones de los integrantes de la Escuela de los Annales, escuela que, como se ha mencionado antes, surge con un marcado interés por resignificar y renovar los métodos históricos tradicionales; en tal sentido, la intención se reconoce entonces que la intención con la que surge la historia regional, se da en virtud de la necesidad de prevalecer el estudio de la historia de regiones específicas y particulares, en cambio de continuar con la vieja tradición de estudiar las grandes civilizaciones.

Las delimitaciones en la narrativa histórica establecidas desde la región van mucho más allá de las construcciones políticas y geográficas y por ello el asunto de las relaciones de frontera es fundamental al pensar la región como un objeto de estudio de la Historia. Algunos docentes de la Universidad de Caldas se han inscrito en esta lógica de pensamiento y excluyen la región histórica de la delimitación exclusiva de los límites político- administrativos actuales. ${ }^{9}$

Una relación de frontera puede estar determinada por una cordillera, o un río, con un entrelazamiento cultural de dos regiones que sí bien, que sí bien, aparentemente son distintas, pueden llegar a tener cosas en comunes como por ejemplo el sistema económico. En este sentido, la Historia Regional hace parte de estudios que tienen que ver con áreas no estrictamente determinadas por fronteras diseñadas en las oficinas de planeación y ordenamiento territorial, sino por las fronteras mismas que diseña la dinámica económica, social, cultural o la misma dinámica política. Las regiones históricas poseen mayor complejidad que las delimitadas en lo político-administrativo y ello se evidencia en la expresión de un docente de la Universidad de Caldas:

Otra perspectiva de la historia regional puede plantearnos el estudio de Suramérica como región, en ese sentido, podemos ver infinidad de situaciones. ¿Dónde se borra la frontera entre Colombia con Brasil? Uno diría que realmente cuando se termina el país, pero no, porque allí la parte cultural juega un papel

\footnotetext{
${ }^{8}$ Desde finales del siglo pasado durante las primeras décadas del siglo XX, el estudio de las regiones y de la relación hombre- espacio- tiempo, experimenta un vuelo importante en su desarrollo, y se convierte en objeto de interés para geógrafos, economistas, sociólogos e historiadores, proceso que está directamente vinculado con el surgimiento de la revista francesa de Annales. A ello se debe la existencia de una amplia gama de criterios y propuestas acerca de las bases teóricas, los métodos y procedimientos para emplear en los estudios regionales y en particular por la historia regional.

${ }^{9}$ Negar los intereses que surgen a partir de los procesos de formación universitaria y la forma en que se consolidan como propuestas que son motivadas por los docentes, sería además de una injusticia en términos académicos y emocionales, un asunto de no reconocer las oportunidades brindadas y que se han convertido en la pauta de proyectos de academia y de vida. Gran parte del interés que motivó esta investigación y que despertó el interés por la Historia Regional a partir de las relaciones de frontera, fue producto de la participación en el proyecto "De epitafios, memorias y rituales: los cementerios como microcosmos simbólico. Estudio en cinco municipios del Eje Cafetero Colombiano", trabajo dirigido en el área histórica por el profesor Luis Fernando Sánchez Jaramillo y en el cual la autora de la presente investigación tuvo la oportunidad de trabajar como auxiliar en el área histórica, recorriendo los municipios seleccionados para el estudio, además del trabajo archivístico e interpretativo.
} 
importantísimo con cuál es la relación que tienen las comunidades de la frontera colombo-brasileña y las comunidades que viven en la frontera brasileñocolombiana, cuál es su cultura, cuál es su relación, cuál es su dinámica, como se desempeñan y qué están haciendo para el desarrollo de esa región, cómo se aporta a un país y se aporta al otro para ver esto desde una dinámica mucho más importante.

Las regiones históricas a partir de las construcciones realizadas por los docentes y desarrolladas por los estudiantes de la Universidad de Caldas, establecen en la Historia Regional una posibilidad de análisis que no excluye las relaciones con otros elementos de la historiografía nacional y narrativas de carácter occidental, pero sí se constituye en una riqueza para nuevas posibilidades sobre las transformaciones culturales y la búsqueda de identidades constructivas. ${ }^{10}$ El objeto de estudio de la Historia Regional es la región, pero la región percibida a través del espacio y del tiempo, a partir de las especificidades, lo cual no quiere decir que no sea una historia interconectada, de interrelaciones con contextos mayores o amplios, en este caso lo nacional y lo universal. La cultura fronteriza hace que los territorios en un momento determinado se unan o desunan en la construcción de cultura bajo diferentes ópticas, entonces el ámbito de la región va mucho más allá de la misma frontera.

\section{Al respecto, vale la pena retomar a Vizcaíno, quien expone que:}

(...) no siempre la historia regional es entendida como tal y suele ser confundida con historia local o simplemente se utiliza el término regional en forma genérica, de manera que contemple estudios relativos a cualquier espacio menor al de un país o nación. A pesar de la frecuencia con que encontramos esta superposición y de su virtual generalización en la historiografía regional, sobre todo en Latinoamérica, es preciso dejar claro que esta analogía es esencialmente falsa. ${ }^{11}$

En esta perspectiva y siguiendo los planteamientos de Vizcaíno, es necesario comprender entonces que, la historia regional tiene un objeto de estudio muy definido y diferente de la historia local, que es la región - como realidad observable a diferentes escalas-; y que dicho objeto de investigación no corresponde, necesariamente, a un espacio pequeño de un país; en tanto es un asunto que puede ser estudiado a diferentes niveles. Es decir, que una región no se circunscribe desde estudios históricos a espacios delimitados por fronteras geográficas, sino en relación a los asuntos a estudiar y que, necesariamente identifican un espacio en un tiempo determinado.

\footnotetext{
${ }^{10}$ Sobre la Historia Regional que se trabaja en la Licenciatura en Ciencias Sociales, de la Universidad de Caldas, dice Sandra Milena Cruz (2007: 115), da elementos tanto históricos como metodológicos para su trabajo en las instituciones educativas de básica primaria y secundaria de la ciudad de Manizales, por tanto, se pretende que el estudiante al finalizar el semestre estudiado, quede con elementos que le propicien una visión más amplia de su región y que también le brinde la posibilidad de hacer historia y utilizar diferentes medios para darla a conocer.

${ }^{11}$ Vizcaíno González, Lilian, "La Historia Regional. Mitos Y realidades" Tzintzun. Revista De Estudios Históricos, 27 (2016): 116-29.
} 


\subsection{Un panorama sobre la enseñanza de la Historia Regional}

Las producciones académicas en la Historia Regional ${ }^{12}$ reconocen una trayectoria importante, además de consolidarse como parte de las academias de historia en diversos municipios y regiones. La necesidad de ecos diversos, narraciones, interpretaciones y sentidos de las sociedades han promovido la creación de centros y grupos de investigación dedicados al estudio del pasado regional. En relación con esta afirmación se puede comprender desde estudios como el realizado por Acevedo:

(...) Para América Latina y especialmente para Colombia los años ochenta significaron el encuentro con la historia regional. Una renovación desde todo punto de vista académico si se tiene en cuenta que la historiografía en nuestro país era todavía una punta ancilar de las historias patrias en las Academias, cuya función no había sido otra que la de legitimar el proyecto republicano independentista a través de una producción heroica y de gesta. La historia regional también entabló un diálogo abierto y de ruptura con aquella historia anclada en las estructuras y superestructuras deterministas del materialismo histórico, cuya tendencia por encontrar modelos teóricos afines y semejantes a los modos de producción asiáticos y europeos, convirtió las historiografías nacionales en recetarios de una profunda fuerza explicativa, pero poco creíbles al contrastarlo con el particular desenvolvimiento histórico de las sociedades que analizaba. ${ }^{13}$

Esta realidad de reconocimientos académicos se vio enmarcada en los procesos de celebración y producción académica del Bicentenario de la Independencia Nacional, pues el proyecto Historia Hoy contó, en su tercera fase, con Historias locales $^{14}$ - memoria plural, resaltando con esto, la necesidad e interés por la pluralidad

\footnotetext{
${ }^{12}$ Una fuerte discusión resulta entre las diferencias y similitudes relacionadas con la historia regional y la microhistoria; por tal razón, es necesario hacer otra precisión (Capdepont, J., 2007:20) “Aunque microhistoria e historia Regional, se proponen entender problemas que son sepultados por las historias nacionales o generales, no son la misma cosa, una se ocupa de lo pequeño y típico y la otra de analizar proceso que afectan a una red social más compleja". De otra parte, es también importante resaltar que, para el caso de la historia regional se reconoce como uno de los principales autores al historiador mexicano Luis González González, dentro de sus obras más reconocidas se destacan: Pueblo en Vilo (1968, 1a edición), Invitación a la microhistoria (1973), Nueva invitación a la microhistoria (1982) y Terruño, microhistoria y ciencias sociales (1991). La microhistoria tiene como uno de sus principales autores al italiano Carlo Ginzburg, de quien se resaltan las siguientes obras: Brujeria y cultos agrarios entre los siglos XVI y XVII(1966), El queso y los gusanos. El cosmos según un molinero del siglo XVI(1976), Pesquisa sobre Piero (1981), Mitos, emblemas, indicios. Morfología e historia (1986), Historia nocturna. Un desciframiento del aquelarre (1989), El juez y el historiador. Acotaciones al margen del caso Sofri (1991), y El hilo y las huellas. Lo verdadero, lo falso, lo ficticio (2010).

${ }^{13}$ Álvaro Acevedo, "La historia regional: un campo abierto de problemas", Revista de Ciencias Humanas UTP, 11., 45 (2005): 100.

${ }^{14} \mathrm{La}$ intención básica de esta fase fue desarrollar investigaciones sobre el qué papel jugó su localidad (vereda, municipio, ciudad, departamento, región, etc.) durante el proceso de la Independencia y cómo se experimentó la construcción de la nación. Para el caso del departamento de Caldas se realizó un trabajo enfocado en la región denominada el Cantón de Supía, que comprende los actuales municipios de Riosucio, Supía, Marmato y Quinchía, este último perteneciente en la actualidad al departamento de Risaralda. También se hicieron diferentes registros fotográficos en los municipios de Manizales y Anserma. En todo este proceso la autora de la presente investigación tuvo la oportunidad de participar y aportar en los escritos, bajo la dirección del profesor Luis Miguel Córdoba de la Universidad Nacional de Colombia,
} 
de la narración histórica regional para analizar diversos procesos y situaciones de la sociedad del pasado y el presente.

Sin embargo, estos esfuerzos mancomunados han tenido poca o casi nula presencia en las instituciones educativas de básica y media en el país, debido a las mismas pautas de los planes de estudios en los cuales se privilegian otros tipos de narrativa y perspectiva sobre el pasado. En atención a ello, Acevedo afirma que,

(...) Lo paradójico de esta renovación académica, que en Colombia adquirió el mote de Nueva Historia, es que no trascendió el cambio de las prácticas pedagógicas en la educación básica y media. Una era la práctica en los programas de historia en las universidades y otra en los manuales y textos escolares, pese a que éstos atendieron a los requerimientos de una reforma integrada de las ciencias sociales. ${ }^{15}$

El tratamiento de las cuestiones regionales es muy inferior a las posibilidades ofrecidas por la legislación vigente, que permite dedicar a estos temas entre un $35 \mathrm{y}$ un 45 por ciento de los contenidos. Por término medio, los contenidos regionales de los distintos manuales de historia no sobrepasan el 10\% de los mismos. Es, por tanto, improcedente y no aceptable, científicamente, afirmar que en los manuales exista un exceso de "localismo". ${ }^{16}$

De otra parte, el mundo de la globalización y la interconexión mundial ha sido otro gran reto para la historia regional, pues este concepto siempre divergente, lo es aún más a partir de las terminologías de aldeas globales, sociedades mundiales e interconexión. En términos de la enseñanza ha sido también un reto para docentes y directivos en aras de las nuevas necesidades de la academia con el concepto de desarrollo propio del mundo globalizado. Su enseñanza a futuro, según Serrano ${ }^{17}$ dependerá de tanto de replantear las situaciones democráticas y sociales de la globalización como del rescate del conocimiento histórico sobre el pasado y presente de las regiones.

Tampoco puede decirse, entonces que el panorama es caótico, pero sí implica una fuerte reflexión sobre las condiciones de formación de los docentes de Ciencias Sociales, los sistemas ideológicos imperantes y la extensión de la producción académica realizada por diversas personas e instituciones dentro del espacio escolar. La producción que existe en los centros de investigación y las universidades es reflejo de una preocupación por lo regional y de esfuerzos mancomunados por otorgar nuevos sentidos y significados a las vivencias de las comunidades. Esta interesante situación contrasta con la realidad de los espacios escolares para la formación en historia, donde

sede Medellín.

${ }^{15}$ Álvaro Acevedo, "La historia regional: un campo abierto de problemas", Revista de Ciencias Humanas UTP, 11., 45 (2005): 100.

${ }^{16}$ Rafael Valls, "La didáctica de la historia en Alemania: una aproximación a sus características". Revista Didáctica de las Ciencias Sociales, Geografía e Historia 21 (1999): 89-105.

${ }^{17}$ Serrano 104 . 
se repiten los enfoques de historias nacionalistas y de corte occidental, los cuales exigen a los maestros en diferentes instituciones y entidades.

Con la intención de superar las posibles tendencias tradicionales de la enseñanza, amparadas en la instrucción de la historia, cimentadas sobre una base de historias de corte nacionalista, importantes académicos de la historia regional en Colombia proponen una relación directa con los procesos de enseñanza y las responsabilidades que tienen tanto historiadores como docentes, en cuanto a las implicaciones éticas de formar en historia en los diversos niveles educativos, allí se puede plantear la necesidad de relacionar las propuestas y avances historiográficos con las implicaciones para la enseñanza:

(...) El crecimiento de la historiografía y la mejor enseñanza de ésta, debe reconocer que los denominados hechos del pasado no son más que reconstrucciones racionales de los mismos para atender a respuestas de nuestro presente y futuro. Puede ser cierto que la historia "matria" de la que nos habla Luis González arremete contra todas las explicaciones al vapor y privilegia la comprensión de los actores del terruño, de la comunidad que construye su propia historia y sus visiones de mundo, pero en uno como en otro caso toda construcción historiográfica es imaginada y está mediada por una visión personal ya sea narrativa o teórica. Ése es el principio de cualquier enseñanza básica en la historiografía. ${ }^{18}$

\section{2. ¿Historia Regional vs Historia Universal?}

El pasado hace parte de cualquier proceso de conformación y consolidación regional, independientemente de las mismas fronteras, precisamente porque esas relaciones culturales son las que hacen que se conformen y consoliden los países; y en dónde, en muchas ocasiones, son las mismas fronteras las que en un momento determinado condicionan la relación de un país con otro; aunque también es posible que la cultura fronteriza permita que las regiones, independiente del país al que pertenecen, en un momento determinado, se unan; aspecto que da la posibilidad de observar una cultura común en varios países o en varios lugares de diferentes países; entonces, es válido reconocer que el ámbito de la región va mucho más allá de la misma frontera.

El objeto de estudio de la historia regional es básicamente analizar todos los procesos sociales y económicos de una región como tal, es decir, todos los aspectos sociales y económicos vistos desde otras perspectivas diferentes a los límites geográficos; desde esta lógica, bien puede decirse que, una visión más amplia permite comprender los principios y razones que orientan la vida de una ciudad, de una población o de una región, entonces, se pude afirmar que desde lo micro, es factible analizar los componentes que dan el desarrollo de una población. La escala de observación básicamente es más micro, es lo que la diferencia con la historia regional y mundial.

${ }^{18}$ Álvaro Acevedo, 105. 
La Historia Regional como cualquier otro tipo de historia puede estudiar todas las acciones de los hombres, entendiendo por acciones de los hombres los eventos y los acontecimientos. Desde ese punto de vista, se puede estudiar la política, la cultura, la economía, la sociedad y todo aquello que tenga relación con todos estos grandes ítems; sin embargo, eso depende de la concepción de historia regional que el historiador tenga. En historia regional es posible historizar todo, entonces no se encuentra ningún inconveniente, porque la historia regional no solamente es una concepción particular de historia, sino que también es una metodología particular para hacer historia; ${ }^{19}$ y lo otro es que de todas esas tendencias del quehacer historiográfico, la tendencia de historia regional es quizás la más pertinente, la más necesaria, la más útil. Y las regiones tienen que empezar por hacer su historia, es decir, de esta no podrán escaparse.

\section{La Historia Regional en Colombia, relaciones desde los Lineamientos Curriculares y Estándares de Ciencias Sociales. Articulaciones con procesos de enseñanza obligatoria}

Para el año 2004, el Ministerio de Educación Nacional, con base en la propuesta de lineamientos Curriculares para la educación básica, publico Estándares Básicos de Competencias en Ciencias Naturales y Ciencias Sociales: Formar en ciencias el desafío; los cuales salieron en solo documento, en tal sentido, puede decirse que existe una importante lógica de fondo, la formación de carácter científico, que no sólo se desarrolla en laboratorios de química o experimentos físicos; sino que otorga a las Ciencias Sociales la responsabilidad de formar en el aprendizaje de los métodos propios de las disciplinas que las conforman.

Si bien es plausible dicha intención, es relevante advertir, que no siempre las apuestas gubernamentales, van en la misma dirección del desempeño y formas de trabajo de los docentes en las aulas; en tanto, es posible encontrar que en varios programas de formación docente se hace poco reconocimiento y además se han desligado gran parte del aprendizaje de las metodologías propias de las escuelas de pensamiento histórico; considerando incluso, en el peor de los escenarios, que la historia ya está hecha, y en tal sentido, en las aula escolares y los educandos que la conforman no se requiere nada diferente a repetirla y memorizarla.

Para el caso de la enseñanza la Historia Regional, de Desde el punto de vista metodológico, tiene una connotación representativa el reconocimiento de nuevas

\footnotetext{
${ }^{19}$ En cuanto a los procesos y formas de trabajo de los historiadores, Scuri, M. y Favaro, O. (2005:3) plantean que, “... sabido es que en la actualidad los historiadores renovamos nuestras explicaciones, modificamos el objeto de estudio, las fuentes y los métodos. Entre los motivos de este cambio está el derrumbe o debilitamiento de los determinismos lineales tanto en el neopositivismo como en el marxismo, en los que subyace una idea de un único sentido de la historia. El historiador puede creer que la historia evoluciona de acuerdo con un progreso incesante sin preguntarse sobre la naturaleza de la misma, los fines o las funciones admitidas o potenciales en las sociedades actuales. Pero si partimos de que no existe un solo motor que impulsa la historia, sino varios, no existe un movimiento continuo y ello sustenta la pluricausalidad.
} 
formas de abordar su estudio y su enseñanza, argumento que permite reconocer la posibilidad de hacer uso y manejo de diferentes fuentes, contrastarlas entre sí y relacionarlas con los cambios y continuidades de las comunidades y, aunque existen estudios que proponen la falta de delimitaciones metodológicas en los estudios de Historia Regional, también es evidente en amplios estudios actuales, que una gran ventaja de la enseñanza de la historia centrada en un enfoque regional, es que en ella se fomenta el acceso a testimonios, registros, edificios, documentos, actas y fotografías, fuentes de información que otorgan al estudiante la posibilidad de relacionar las posturas teóricas con las interpretaciones fruto de procesos y procedimientos de la historia.

Lo anterior se relaciona directamente con percepciones de docentes de Historia en la Licenciatura en Ciencias Sociales, quienes reconocen que los docentes son actores vitales en el proceso de formación, entonces ellos tienen que conocer obligatoriamente la Historia Regional, pues de los contextos parten intereses para cambiar, mejorar, construir y deconstruir la región, entonces es fundamental una formación no sólo conceptual sino procedimental sobre las posibilidades de la enseñanza de la Historia y reconocen en la perspectiva regional una gran opción.

Al hacer referencia al aspecto procedimental, se puede establecer una relación con los estándares de Ciencias Sociales (2004), particularmente en uno de los ejes básicos: aproximación al conocimiento como científico y científica social, tipos de desempeño que son reconocidos en dicho documento como un pilar fundamental de la formación por competencias. Allí no sólo es importante, sino necesario el acercamiento y el manejo de fuentes históricas, geográficas y demográficas desde los contextos de los educandos, lo cual se puede evidenciar en la siguiente tabla, que retoma algunos elementos presentes en los Estándares para la formación en Ciencias Sociales:

Tabla 1. ejemplos de indicadores de desempeño en Ciencias Sociales

Grados

\section{Ejemplo de indicador de desempeño}

Primero a Tercero

Reconozco diversos aspectos míos y de las sociedades a las que pertenezco, así como los cambios que han ocurrido a través del tiempo.

Cuarto a Quinto

Utilizo diferentes tipos de fuentes para obtener la información que necesito.

Sexto a Séptimo

Recolecto y registro sistemáticamente información que obtengo de diferentes fuentes.

Octavo a Noveno

Hago planes de búsqueda que incluyan posibles fuentes primarias y secundarias.

Décimo a Once
Realizo investigaciones como lo hacen los científicos sociales: diseño proyectos, desarrollo investigaciones y presento resultados.

Fuente: elaboración propia a partir de los Estándares de Ciencias Sociales (MEN, 2004) 
El cuadro anterior, realizado a partir de elementos presentes en el documento del MEN y que rige desde el año 2004 en las instituciones educativas públicas y privadas del país, resalta un eje temático que incluye directamente el desarrollo de elementos regionales a partir del manejo de fuentes y la identidad de grupos. Esto, pareciese no tener relación con los elementos propios de la Historia Regional, pues en ningún momento se refiere de manera directa a este tipo de estudios. Sin embargo, de manera inferencial es importante reconocer que, para un adecuado manejo de fuentes primarias, es imprescindible tener algún tipo de cercanía a las mismas y, por tanto, ello implicaría abordajes desde la región, ya sea desde la demografía, la geografía o las narrativas históricas. Esta situación resulta complicada sí se relaciona con elementos de Historia Universal, ante los cuales las fuentes posibles para manejar en el aula de clase son casi en su totalidad secundarias, pues dependen del acceso a las mismas. Ante esto la profesora de la Universidad de Caldas, Molina, plantea:

(...) A través de la historia regional descubrimos también que todos tenemos historia, que podemos reconstruir la historia de aquellas personas, villas, comunidades o sociedades que nunca la han tenido (...) Podemos ejemplificar, para adentrarnos en una experiencia histórica regional, a la historia oral como un método, a la entrevista como una técnica y al cuestionario como instrumento. ${ }^{20}$

Ahora bien, es claro que la formación como científicos históricos y sociales en la educación básica y media, no tiene como fin profesionalizar al estudiante en estas áreas del saber, sino sensibilizar, potencializar y formar desde la diversidad posible, en las comprensiones del pasado y evidentemente las repercusiones en las construcciones sociales de las que él, ella y ellos hacen parte. Formar para el conocimiento científíco de las Ciencias Sociales tiene entonces, como intención sensibilizar al estudiante sobre el dinamismo del saber propio del área y las metodologías pertinentes para interpretar las comunidades a través del tiempo y el espacio. Este acercamiento tiene mayor sentido desde y por los contextos socialmente significativos para los educandos y en los cuales pueden realizar los ejercicios de manejo e interpretación de fuentes, además del cuidado y responsabilidad social de las mismas, aportando a los elementos de formación para el patrimonio, al respecto un docente del programa de Ciencias Sociales de la $\mathrm{U}$ de Caldas dice:

... un asunto interesante de los lineamientos curriculares para el área de Ciencias Sociales y particularmente en lo referente a la enseñanza de la Historia, es que permite a los estudiantes tener acercamiento a fuentes de información primarias, que les brinden herramientas para interpretar la historia de su contexto; es decir, cambia esa lógica de enseñanza que se centra primordialmente en transmitir información de los hechos que otros historiadores han logrado plasmaren libros reivindicando el papel de los héroes de la Patria. Esta nueva lógica de la enseñanza propuesta de los lineamientos y las competencias, lo que promueve es un escenario educativo en el que los estudiantes pueden aprender desde la experiencia cómo se construye conocimiento histórico; en tanto los lleva a comprender la historia desde su contexto político, cultural, económico y social cercano y desde el rescate de la identidad regional.

\footnotetext{
${ }^{20}$ Mercedes Molina, "Historia regional y microhistoria. Necesidad Grancaldense", Revista Latinoamericana de Estudios Educativos, 2., 2 (2006): 155.
} 
La sociedad reclama sin duda la formación de grupos con mayor nivel de análisis de su sociedad, que comprendan cómo la política impregna todos los espacios de la vida humana, donde exista articulación entre la crítica y las opciones de crecimiento y transformaciones sociales. Allí las Ciencias Sociales como área formal de la enseñanza obligatoria deben responder a las comunidades, pues ¿cuál es la intención de que un ser humano se comprenda como ser en el tiempo y en el espacio? Y ¿Qué intención de saber tiene incidencias en la vida de un ciudadano o ciudadana del mundo?

La Historia como ciencia de los hombres en el tiempo es transversal a la vida de cada individuo dentro de su contexto y por esto analizar la conciencia histórica como eje de la formación del futuro docente requiere tener en cuenta la complejidad de la cultura humana, que sólo se explica a través de los cambios y continuidades. La sensibilización de los científicos sociales en la escuela requiere de compromisos con los contextos por parte de las comunidades e involucran en sí mismos a los futuros educadores y la formación procedimental que aprenden en sus estudios universitarios.

Los elementos anteriormente mencionados, permiten identificar, que quizá de diversas maneras se aborda la Región y la Historia Regional es escenarios de clase, pues el acercamiento a lo local, lo barrial, a la ciudad y los elementos formativos que se construyen desde este, pueden visibilizar de manera más clara, que docentes, instituciones y procesos de investigación, pueden fundamentarse en las dinámicas regionales desde múltiples perspectivas, sea de manera integrada o fragmentada entre las Ciencias Sociales, con relaciones hacia otras áreas de la enseñanza obligatoria. También es importante reconocer la diversidad de investigaciones regionales que existen dentro de los grupos de investigación y las universidades, pero como se mencionó desde el inicio del trabajo, la intención de esta propuesta es establecer los puntos de encuentro y distancia con los procesos de la enseñanza en la educación básica y media colombiana, a partir de los procesos formativos de los futuros docentes del campo profesional.

\section{Historia Regional para la formación docente: caso y realidad nacional- Universidad de Caldas}

Los planes de estudio para los programas de formación de docentes en Ciencias Sociales y en Historia del país, ${ }^{21}$ excluyen de manera directa en sus procesos de formación el carácter obligatorio de la formación en Historia Regional, en las universidades ofertadas su condición es como asignatura electiva, con excepción del programa de Licenciatura en Historia de la Universidad del Valle, en el cual se incluyen los cursos de Historia Oral e Historia del suroccidente colombiano. Sin embargo, este análisis no pretende afirmar la inexistencia de cualquier tipo de Historia Regional en la formación de los licenciados, pues a partir de cursos como archivística o paleografía se trabaja sobre los documentos de los archivos municipales

\footnotetext{
${ }^{21}$ Allí se incluyen todas los programas que fueron analizados durante el capítulo uno de la presente investigación. Es decir, Licenciaturas en Ciencias Sociales, Licenciaturas en Educación Básica con énfasis en Ciencias Sociales y Licenciaturas en Historia.
} 
y departamentales, además de los manejos internos que desde la libertad de cátedra puedan otorgar los docentes a sus cursos dentro de los programas.

El asunto que sí puede afirmarse, es que en los distintos niveles de educación y, de igual manera en los planes de estudio de los programas de formación de educadores en Ciencias Sociales y en Historia, la principal preocupación ha sido dar prioridad a los cursos de historias de corte occidental, latinoamericana y nacional dentro de los planes de estudio de las universidades, posiblemente en aras del cumplimiento a los indicadores conceptuales básicos propuestos por la legislación educativa y la necesidad de unificar elementos para las pruebas externas que se aplican de manera igual a todos los estudiantes del país. ${ }^{22}$ En este sentido, al docente hay que formarlo con lo que serán sus herramientas en el campo de acción y en la competitividad del mercado laboral; esta afirmación es válida, más no del todo cierta, pues como se analizó en el ítem anterior, la misma legislación vigente articula elementos de carácter procedimental que son importantes para la formación en Ciencias Sociales y por tanto deberían ser objeto de reflexión en los programas de licenciatura de las universidades.

Para el caso de la Universidad de Caldas, ${ }^{23}$ la Historia Regional, que se aborda en el programa como una asignatura electiva; otorga algunos elementos históricos y metodológicos para el trabajo en las instituciones educativas de básica primaria y secundaria de la ciudad de Manizales, por tanto, se pretende que el estudiante al finalizar el semestre, en el cual cursa dicha asignatura, "quede con elementos que le proporcionen una visión más amplia de su región y que también le brinde la posibilidad de hacer Historia y utilizar diferentes medios y darla a conocer". ${ }^{24}$

Dentro de la justificación del programa puede identificarse un compromiso de carácter regional a partir de un campo de acción: "Formar un nuevo educador, que además de su rol de docente, pueda desempeñarse como promotor de cambios socioeconómicos, en los contextos nacional y regional, y que aporte desde la investigación soluciones al desarrollo no sólo de la educación sino en el fortalecimiento de la democracia y las diversas dinámicas sociales". Esta premisa involucra de

\footnotetext{
${ }^{22}$ Hay tres asuntos que, según Rafael Valls (2011), seguramente han hecho que se piense en una enseñanza de la historia más universal y general. Por una parte, la reticencia, incluso rechazo, de las innovaciones didácticas, que habían intentado superar un tipo exclusivamente transmisivo de la enseñanza de la historia mediante nuevas formulaciones en las que el alumnado tuviese una participación más activa en el proceso de enseñanza y de aprendizaje, que en muchas ocasiones se estaba acompañando de un tipo de enseñanza vinculado al estudio escolar de los procesos históricos y de los problemas relevantes más conectados con el presente. Por la otra, su desacuerdo con la forma en que se había realizado la inclusión de las historias regionales o locales en los programas escolares, por considerar que ocupaban un excesivo espacio dentro de la enseñanza de la historia y que, además, perturbaban una comprensión unitaria de la historia del país y finalmente, la consideración de que la historia contemporánea tenía un excesivo protagonismo en el conjunto de los programas escolares, especialmente en el Bachillerato.

${ }^{23}$ Los fundamentos para la construcción de este apartado hacen parte de la tesis de maestría de la profesora Sandra Milena Cruz Osma, titulada "Enseñanza de la Historia Regional en la Licenciatura en Ciencias Sociales de la Universidad de Caldas" y que consolidó de igual manera un artículo para la Revista Latinoamericana de Estudios Educativos (2008).

${ }^{24}$ Sandra Cruz, "Enseñanza de la Historia Regional en la Licenciatura en Ciencias Sociales de la Universidad de Caldas, Colombia. Revista Latinoamericana de Estudios Educativos, (2007): 115.
} 
manera directa el asunto por y desde lo regional, comprendido tradicionalmente para el caso de Caldas como el gran sistema del Eje Cafetero y Antioquía, pero que incluye también elementos del Cauca como parte de la construcción de la identidad para algunos territorios del departamento.

En virtud de lo anterior, que se deduce del propósito del programa, Cruz considera que “... se hace evidente la necesidad de conocer la región, llegar a aportar y generar conocimiento, en un futuro, a la sociedad caldense y colombiana; conocimiento que no solo será para desarrollarlo en las instituciones científicas del país, también en las de carácter educativo". 25

Otro aspecto relevante que se halló en la revisión documental, es que en el pénsum académico desarrollado antes del año 2002, aparecía la asignatura: Problemas socio geográficos de Caldas y Manizales, allí se desarrollaban aspectos geográficos e históricos de la región del Viejo Caldas, a través de salidas de campo, en las cuales se procuraba conocer características arquitectónicas, culturales y sociales de los pueblos y veredas de la región, a partir del 2002 la asignatura cambia de nombre, Geografía de Caldas y Manizales, teniendo en cuenta prácticamente los mismos planteamientos del programa académico de área anterior.

La enseñanza de la Historia Regional en la Licenciatura en Ciencias Sociales hace parte, como se mencionó anteriormente, de las asignaturas electivas que los estudiantes pueden cursar según sus gustos y afinidades, pero que no constituye un requisito de grado para el futuro docente. El recorrido marcado por esta asignatura se identifica por un trayecto desde el conocimiento de geografía, pasando por el estudio de sus diversas familias indígenas de la región, el proceso de mestizaje, las diferentes transformaciones sociales del siglo XIX, la Colonización Antioqueña, la conformación del nuevo departamento de Caldas, transformaciones en el paso de campo a ciudad, conocimiento de la identidad regional, a través de la evolución histórica nacional, hasta la importancia de la historia regional para América Latina, Colombia y Caldas, temas que han ayudado a los estudiantes a ampliar un poco la visión sobre el territorio que habitan.

El interés y la preocupación por el tema de región es una situación que relaciona diversas áreas del saber, donde convergen la geografía, la economía, la política y la demografía. Estas múltiples miradas pueden generar, en el desarrollo del curso, un problema en el momento de definir y conceptualizar qué es, qué identifica y qué compone una región; discusión a la cual se convoca a la historia regional y que hace complejo delimitar el campo de estudio propio, así como las metodologías de trabajo que las caracterizan.

Aunque los Lineamientos Curriculares de las ciencias sociales establecen la necesidad de formar para las relaciones globalidad-localidad y la identificación con los problemas de las comunidades; la realidad es que la historia regional está

${ }^{25}$ Cruz 117. 
casi eliminada de los planes de estudio en las instituciones educativas, con excepción de aquellos casos en los cuales la trayectoria cultural del municipio tiene un interés representativo por los valores históricamente adquiridos y que representan a la comunidad. Para el caso del departamento de Caldas se destacan fundamentalmente las cátedras municipales en Riosucio, Anserma o Salamina, algunas de ellas cimentadas por la declaratoria de Paisaje Cultural Cafetero. ${ }^{26}$

La región estudiada en la escuela ha sido un concepto particularmente abordado por la geografía y por tanto se desliga de comprensiones y elementos históricos que permitan un análisis integrado en aras de la interdisciplinariedad que se busca en las Ciencias Sociales. El asunto no está en obligar a cursar o no una asignatura de Historia Regional, sino en la necesidad de replantear algunos elementos de carácter formativo en Ciencias Sociales que trascienden del asunto conceptual e involucran de diversas maneras el asunto y la preocupación por la región, pues la producción realizada por historiadores e investigadores debe aportar de algún modo en los espacios de la enseñanza básica y media, en aras de sensibilizar, acercar y aportar desde la escuela a los sentidos de región y la comprensión de identidades plurales y necesarias en el contexto colombiano. Al respecto, vale la pena traer la expresión de Molina, quien expresa de manera concreta la idea propuesta: "El conocimiento histórico se construye para ser difundido, en este caso, a la juventud que se encargará de compartirlo; igualmente para propiciar investigaciones interdisciplinarias de carácter regional, con el fin de saber de dónde y de quiénes procedemos". ${ }^{27}$

\section{1. ¿Para qué enseñar desde la Historia Regional? Abordaje de la investigación}

Para responder este cuestionamiento es importante recordar las nociones de Santos (1990) para referirse a las relaciones espacio-tiempo y abordar de esta manera lo regional como perspectiva histórica con múltiples posibilidades, puesto que el conocimiento del espacio como categoría universal se incluye en el conocimiento del espacio como categoría histórica y viceversa.

Pero el espacio no es vivido ni sentido de la misma manera por todas las comunidades, pues la experiencia temporal está delimitada por elementos de carácter cultural que hacen parte de la memoria y la historia incorporada, permitiendo poner las formas y expresiones de las memorias que se llevan dentro. La relación con la memoria está atenta a la relación del individuo como actor social con el mundo cultural que lo contiene y también al trabajo sobre ese objeto de estudio, o sea a las alusiones

\footnotetext{
${ }^{26}$ El Paisaje Cultural Cafetero Colombiano (PCCC) es un paisaje cultural y productivo en el cual se combinan elementos naturales, económicos y culturales con un alto nivel de homogeneidad en la forma de plantaciones cafeteras y sus paisajes. Pocas décadas tras la arribada de los colonos en Antioquia en el siglo XIX, la economía y la cultura de la región se desarrolló alrededor de una tradición cafetera con fuertes raíces, la cual a dejado un legado único definiendo la cultura regional, con ricas manifestaciones en la música, comida, arquitectura, y educación. Las características del PCCC son el resultado del proceso de adaptación de estos colones, un proceso que perdura hasta el día de hoy.

${ }^{27}$ Mercedes Molina, "Historia regional y microhistoria. Necesidad Grancaldense", Revista Latinoamericana de Estudios Educativos, 2., 2 (2006) 153.
} 
que hace la historia incorporada sobre los contenidos de ese mundo colectivo que alberga el individuo. Por la historia incorporada se aprehende el mundo social bajo las posturas de Bourdieu y se realizan los ejercicios de la memoria por los cuales se rutiniza e incorpora la cultura (Nates, Jaramillo, Hernández, 2004).

Pensar en las regiones como objeto de estudio histórico, implica desestructurar el límite político o natural, pues la complejidad de las relaciones humanas y las dinámicas que otorgan al espacio en las construcciones culturales, no tienen sentido de la misma manera que las regiones establecidas por los estamentos de planeación nacional ni las divisiones territoriales producto de decisiones administrativas de los gobiernos. Tal como lo plantea Santos: “estudiar una región significa penetrar en un mar de relaciones, formas, funciones, organizaciones, estructuras, con sus más diversos niveles de interacción y contradicción" (1996, 46). El siguiente ejemplo expresado por un docente de la Licenciatura en Ciencias Sociales permite comprender de manera concreta la afirmación de Santos:

La perspectiva de las Europas, en el caso de la Europa Occidental, Europa Central, Europa Oriental; posee una riqueza de situaciones cultuales, económicas, políticas, religiosas que desarrollan la Historia Regional, en tanto que no debemos tener como epicentro de los desarrollos ni de Francia o Inglaterra o Moscú, pues esta división no se refiere a países sino a regiones. Por ejemplo, los eslavos una región supremamente interesante que han hecho un aporte gigantesco al asunto del desarrollo de todo lo que ha sido históricamente Europa Oriental, inclusive desde el siglo IX cuando nace la gran Rusia es una región de muchísimo interés y en ese sentido uno mira la región en otro contexto.

Esta referencia, que es sólo una ejemplificación de las muchas que podrían realizarse, permite identificar la complejidad al abordar la región en términos históricos y la riqueza de estos estudios como posibilidad de comprender las transformaciones de los humanos a través del tiempo y el espacio, como proceso donde lo único constante es el cambio y que establece relaciones de cercanía o distancia con territorios, que aunque separados o unidos desde las directrices político-administrativas, presentan elementos culturales y determinan por ende percepciones, ideologías y prácticas de vida.

Otro caso que podría resultar interesante dentro de esta concepción dialéctica de las regiones fronterizas es el que identifica la conformación cultural del denominado Gran Caldas, ${ }^{28}$ pues, aunque los intentos generalizadores de las narrativas históricas

\footnotetext{
${ }^{28}$ En términos generales se puede definir el Gran Caldas o Viejo Caldas como la toponimia con la cual se delimitan los territorios actuales que identifican de los departamentos de Risaralda, Caldas y Quindío, aunque según estudios de históricos regional muestra que se integra el sector del norte del Valle del Cauca. Esta vasta región que hoy agrupa al antiguo Caldas y norte del Valle existen muchas zonas con profundas diferencias entre sí (norte de Caldas, región minera, resguardos indígenas, zona cálida del oriente, valle del Risaralda, el Quindío, norte del Valle y otras), hubo numerosos factores que contribuyeron a darle unidad económica, social y cultural a la región: las vías de comunicación, el comercio y el café, entre otros. De este modo el desarrollo de las fuerzas productivas, como consecuencia del fenómeno de colonización, produce
} 
le han circunscrito exclusivamente como parte de los procesos de Colonización Antioqueña ${ }^{29}$, al analizar las relaciones de frontera con la conformación geográfica y cultural de los territorios, es interesante comprender las situaciones de poblaciones dentro de procesos de colonización caucana, tolimenses o de las zonas del altiplano cundiboyancese, lo cual relaciona la historia de la gran región caldense como parte de un territorio diverso, pluriétnico y pluricultural, que debe ser estudiado más allá de los procesos de colonos antioqueños, sin decir que estos no sean importantes o representativos dentro de las narrativas históricas.

Siguiendo a Santos, podría concebirse entonces que el mismo dinamismo regional como parte real de la vida humana se desarrolla a través de diferentes tipos de duración y está sujeta a permanentes posibilidades y transformaciones del entorno como acción intencional de las comunidades humanas: El paisaje es formado por los hechos del pasado y el presente. La comprensión de la organización espacial, como de su evolución, sólo se torna posible mediante una cuidadosa interpretación dialéctica entre las formas, la estructura y las funciones a través del tiempo.

Estas posturas implican como hecho vital pensar en relaciones regionales, que no niegan la posibilidad de comprender otros aspectos de la historicidad, pero permiten otras posibilidades de sentido. La globalización como fenómeno innegable para la mayor parte de las comunidades del planeta, ha permitido la creación de conciencias cada vez más particulares de sociedad y modos de vivir, en aras de las mismas búsquedas de expresiones de identidad para los sujetos. Estas relaciones se ven sujetas con la memoria y ante ello puede comprenderse la afirmación de Prats:

Para que la llamada Memoria Histórica llegue a ser provechosa e inspiradora de aprendizajes, el requisito fundamental es que sea verificada y pensada a través de la historia... Trabajar la memoria histórica con los estudiantes un reto de gran interés didáctico: la combinación de lo micro y lo macro considerados holísticamente... ${ }^{30}$

En la dinámicas de los regional y lo local se desarrollan las dinámicas de construcción identitarias (Fernández 2007). La región es entonces el efecto de un proceso de estructuración social que articulando tiempo y espacio concentraría disimiles procesos sociales, este conjunto implica una territorialización de las relaciones histórico-sociales. El espacio, es el espacio de cada tiempo, el espacio como categoría histórica es el propio de los objetos, su significado, su contenido y las relaciones entre ellos que mudan con la historia.

diferenciación y especialización de las actividades, lo que conduce al mercado de aldea y al crecimiento de los pueblos integrando la región a la vida nacional. (Valencia, 2000).

${ }^{29}$ La Colonización antioqueña fue un fenómeno expansivo de población que marcó un hito social y económico en el siglo XIX, implicó la aparición y fundación de centros urbanos y la consolidación de lo que hoy conocemos como el eje cafetero. Geográficamente, esta expansión hizo que antiguos Departamentos como Antioquia y el Gran Caldas dieran lugar a nuevos Departamentos y el crecimiento poblacional de otros. Las zonas que se vieron influidas por el crecimiento poblacional fueron Chocó, Norte del Valle del Cauca y parte del Tolima.

${ }^{30}$ Prats 35 . 
La era de la globalidad es también el reto por la era de la localidad, de la región, de crear nuevas interconexiones que permitan también reconocer relaciones históricas entre lugares y contextos que antes podían parecer imposibles de afectarse mutuamente. Esta situación no puede negarse en la enseñanza de las Ciencias Sociales y de la Historia, pues permiten otorgar nuevos sentidos a su enseñanza dentro del reto en la formación de "ciudadano global", término basado en las ideas sobre ciudadanía de Adela Cortina (1997) y que a su vez es un puente entre los múltiples significados culturales que debe otorgar la globalización, fenómeno que supera lo netamente económico.

Este caso es representativo en un territorio como el colombiano, pues Colombia es un país de regiones, Colombia es un país pluriétnico y pluricultural no es un país de departamentos, Colombia es un país de región, pero la región cultural, la región económica, digamos por esa región que podría denominarse étnica podría denominarse dentro de estos procesos de consolidación de un país entero. El respeto y la valoración de las regiones como integradoras de los lugares de existencia, los cuales no sólo se estudian como operación intelectual, sino como hecho sensible permiten comprender y relaciones los lugares de memoria (Halbwachs), con los espacios que se construyen en el sentir, el pensar y el vivir de los sujetos y que hacen parte de su identidad impuesta o también de su identidad consciente.

Se podría afirmar que en Historia Regional es posible historizar todo, porque la historia regional no solamente es una concepción particular de historia, es una metodología particular para hacer historia y en estas tendencias del quehacer historiográfico podría convertirse en una de las más pertinentes, necesarias y útiles para los historiadores, demás científicos sociales y docente, pues en palabras de docentes de la Universidad de Caldas: "las regiones tienen que empezar por hacer su historia, es decir, de esta no podrán escaparse."

\section{Conclusiones}

Las concepciones de docentes sobre historia regional se movilizan principalmente hacia una función orientadora y crítica, que utiliza el saber propio de las regiones en aras de generar identidad en las comunidades otorgando un sentido diferente a la enseñanza de la historia. Este proceso se señala como complejo desde las diversidades de asignaturas y contenidos estudiados por los futuros docentes a lo largo de su formación y las pocas relaciones de construcción académica generadas entre los departamentos encargados de ofertar los cursos fundamentales de los estudiantes. Esto podría explicar que los conceptos tienen amplias reflexiones sobre las relaciones reales entre pasado-presente-futuro desde los contextos regionales y cercanos

Conceptualizar la región y la Historia Regional como una manera de abordar la historia de los contextos ha dado la emergencia de dos importantes posturas: la primera de ellas es el reconocimiento de la región histórica como un concepto que va más allá de la producción historiográfica del contexto manizaleño, caldense o cafetero, situación que amplía los análisis regionales hacia otros territorios por fuera 
de la regionalización tradicional y posibilita el estudio de los contextos en diferentes áreas del saber propio de la Licenciatura y su ejercicio docente. Como segundo punto, se establece una posibilidad de ver la región histórica como una forma de desarrollar procedimientos propios de la historia, reto enmarcado en los Lineamientos Curriculares para las Ciencias Sociales y que otorgan al docente la posibilidad de relacionar la región en las aulas de clase, además en una relación con otros enfoques de la historia, pero que no niegan las posibilidades de estudios dinámicos sobre las relaciones de frontera y la relación de las comunidades en las mismas.

Enseñar historia, siguiendo a Rüssen tiene una función orientadora en la vida humana, nadie dice hacia dónde o cómo pues esto sería un determinismo incoherente con la postura crítica de las ciencias sociales y la historia. El asunto es comprender y aceptar la condición innegable de temporalidad que poseen los humanos, tanto aquellos que algunas vez llegan a estudiar la historia profesionalmente como aquellos que no; la enseñanza de la historia debe responder a ambas situaciones y contextos, por tanto, aportar a la condición del ciudadano, que debe trascendentes de la mayoría de edad de kantiana, para convertirse en un pilar de la formación humana.

Narrar, contar y aprender sobre el pasado de cualquier grupo humano implica un saber de carácter espacial, pues los sujetos se desenvuelven en un territorio en el que tienen la posibilidad de cambiar conscientemente. Por ello, cualquier historia es una historia del espacio a través del tiempo y del tiempo a través del espacio. Esta situación es quizás uno de los pilares que sostienen el estudio de las Ciencias Sociales en su carácter interdisciplinario y cuyos ejes centrales son la Historia y la Geografía.

Puede plantearse que los sentidos sobre el tiempo no pueden comprenderse sin percepciones de espacio y sobre todo en los espacios cotidianos para los sujetos y su entorno social, esos lugares que son vividos en la cotidianidad y que poseen en sí mismos un significado histórico y un trasegar a través del tiempo. Estas preocupaciones son pilares para el estudio de la Historia Regional, un campo de pensar y construir la historia que trata de regiones, de espacios en los cuales los grupos o instituciones cobran conciencia sobre la pertenencia de los individuos a una etnia, comunidad cultural o población, propiciando la integración y perduración del grupo como colectividad.

\section{Bibliografía}

\section{Fuentes primarias}

\section{Entrevistas}

Entrevistas a docentes en ejercicio y formación, Licenciatura en Ciencias Sociales, Universidad de Caldas. Julio-septiembre de 2011. 


\section{Fuentes secundarias}

\section{Libros}

Cortina, Adela. Ciudadanos del mundo, hacia una teoría de la ciudadanía. Madrid: Alianza Editorial, 1997.

Nates, Beatriz., Jaramillo, Pablo y Hernández, Gregorio. Más allá de la historia, sentidos de pertenencia, socialización y economía en el concepto de pueblo de los Andes. Manizales, Colombia: Editorial Universidad de Caldas, 2004.

Santos, Milton. Metamorfosis del espacio habitado. Barcelona, España: Editorial Oikos-tau , 1996.

\section{Capítulos de libros}

Valls, Rafael. "La enseñanza de la historia: entre polémicas interesadas y problemas reales” En: Miradas a la historia: reflexiones historiográficas en recuerdo de Miguel Rodríguez Universidad de Murcia, 2004.

Van Young, Eric, "Haciendo historia regional: Consideraciones metodológicas y teóricas” En: Pérez, P. Comp. Región e historia en México (1700-1850): Métodos de análisis regional, 99-122). México: Instituto de Investigaciones José Luis Mora Universidad Metropolitana, 1987.

\section{Artículos de revistas}

Acevedo Tarazona, Álvaro. "La historia regional: un campo abierto de problemas", Revista de Ciencias Humanas UTP, 11.45 (2005): 99-108.

Cruz Osma, Sandra Milena. "Enseñanza de la Historia Regional en la Licenciatura en Ciencias Sociales de la Universidad de Caldas, Colombia", Revista Latinoamericana de Estudios Educativos (2007): 111-131.

Fernández, Sandra. "Tras las huellas de lo local y lo regional: notas críticas y tendencias del análisis en la historiografía Argentina”. Revista Historia da historiografía 3 (2009): 161-166.

Miño, Manuel. “Existe la historia regional?” Revista de Historia Mexicana 2.4 (2002).

Molina, Mercedes. "Historia regional y microhistoria. Necesidad Grancaldense". Revista Latinoamericana de Estudios Educativos, 2.2 (2006); 149- 166.

Prats, Juaquín. "Dificultades para la enseñanza de la Historia en la educación secundaria: reflexiones ante la situación española. Revista de teoría y didáctica de las Ciencias Sociales, 5 (2000): 1-11. 
Tejidos de Clío construidos en regiones que transforman el espacio a través del tiempo ...

Rüssen. J. (2006). Didáctica da historia: pasado, presente e perspectivas do caso alemao. Ponta Grossa, Portugal: Editoral Praxis educativa 1.2 (2006): 1-14.

Valls, Rafael. "La didáctica de la historia en Alemania: una aproximación a sus características". Revista Didáctica de las Ciencias Sociales, Geografia e Historia número 21 (1999): 89-105.

Vizcaíno González, Lilian. "La Historia Regional. Mitos Y realidades”. Tzintzun. Revista De Estudios Históricos, 27 (2016): 116-29.

\section{Tesis, ponencias y otras publicaciones}

Boisier, Etcheverry. "Territorio, Estado y Sociedad en Chile, la dialéctica entre la descentralización: entre la geografía y la gobernabilidad”. Tesis doctoral, Universidad de Alcalá. Alcalá de Henares, España, 2007.

\section{Publicaciones en Internet}

https://www.mineducacion.gov.co/ 